\title{
The Cultural Transmission of Prestige and Dominance Social Rank Cues: an Experimental Simulation
}

\author{
Ángel V. Jiménez ${ }^{1}$ (D) Alex Mesoudi $^{1}$
}

Received: 6 July 2020 / Revised: 19 August 2020 / Accepted: 20 August 2020 / Published online: 30 September 2020

(c) The Author(s) 2020

\begin{abstract}
Informal social hierarchies within small human groups are argued to be based on prestige, dominance, or a combination of the two (Henrich \& Gil-White, 2001). Prestige-based hierarchies entail the ordering of individuals by the admiration and respect they receive from others due to their competence within valued domains. This type of hierarchy provides benefits for subordinates such as social learning opportunities and both private and public goods. In contrast, dominance-based hierarchies entail the ordering of individuals by their capacity to win fights, and coerce or intimidate others. This type of hierarchy produces costs in subordinates due to its aggressive and intimidating nature. Given the benefits and costs associated with these types of social hierarchies for subordinates, we hypothesised that prestige and dominance cues are better recalled and transmitted than social rank cues that do not elicit high prestige or dominance associations (i.e. medium social rank cues). Assuming that for the majority of the population who are not already at the top of the social hierarchy it is more important to avoid the costs of dominance-based hierarchies than to obtain the benefits of prestige-based hierarchies, we further hypothesised that dominance cues are better transmitted than prestige cues. We conducted a recall-based transmission chain experiment with 30 chains of four generations each $(N=120)$. Participants read and recalled descriptions of prestigious, dominant, and medium social rank footballers, and their recall was passed to the next participant within their chain. As predicted, we found that both prestige cues and dominance cues were better transmitted than medium social rank cues. However, we did not find support for our prediction of the better transmission of dominance cues than prestige cues. We discuss whether the results might be explained by a specific social-rank content transmission bias or by a more general emotional content transmission bias.
\end{abstract}

Keywords Prestige $\cdot$ Dominance $\cdot$ Status $\cdot$ Social rank $\cdot$ Cultural evolution $\cdot$ Social learning

\section{Introduction}

Social hierarchies are a universal phenomenon in our species (Von Rueden 2014), emerging rapidly and spontaneously during social interactions (Anderson \& Kilduff 2009; Cheng et al. 2013; Smith \& Foti 1998). Being at the top of

Electronic supplementary material The online version of this article (https://doi.org/10.1007/s40806-020-00261-x) contains supplementary material, which is available to authorized users.

Ángel V. Jiménez

aj419@exeter.ac.uk

$\triangle$ Alex Mesoudi

a.mesoudi@exeter.ac.uk

1 Human Behaviour and Cultural Evolution Group (HuBCEG), Department of Biosciences, University of Exeter Cornwall Campus, Penryn, Cornwall TR10 9FE, UK the hierarchy in a human social group is associated with positive fitness outcomes such as greater access to resources, mating opportunities, and greater number of surviving offspring (Betzig 1988; Chagnon 1988; Hill 1984; Mealey 1985; Savin-Williams 1979; Snyder et al. 2008; Von Rueden 2014; von Rueden et al. 2010; von Rueden \& Jaeggi, 2016). People at the top usually act as leaders of groups, which helps to solve group problems such as collective decisionmaking and within-group coordination (Anderson \& Willer 2014). Consequently, social hierarchy is not only beneficial for the individuals at the top but also, potentially, for groups and their members. Nevertheless, hierarchies also produce costs for groups and their members as they can lead to abuse of lower social rank individuals, which diminishes group morale and lowers group performance (Anderson \& Willer 2014). 
The dual evolutionary model of social hierarchy (Cheng 2019; Cheng \& Tracy 2014; Cheng et al. 2013; Henrich \& Gil-White 2001; Jiménez \& Mesoudi 2019b; Maner \& Case, 2016; Redhead et al. 2018a) might help to explain this contrast between the benefits and costs of social hierarchy. According to this model, informal social hierarchies within small human groups can be based on prestige, dominance, or a combination of the two. Prestige-based hierarchies entail the ordering of members of a social group by their prestige (sometimes referred to as "status"), which is defined as the respect, esteem, and admiration that they receive by other members of the group (Anderson et al. 2015). Prestige is voluntarily given by others (Blader \& Chen 2014; de Waal-Andrews et al. 2015), usually due to being competent in domains that are appreciated by a group (Henrich \& Gil-White 2001). For example, frequently scoring goals is an indicator of competence in football, which is highly regarded by people who play or like football. As such, top goal scorers tend to attain prestige within football teams. However, competence might often not be enough to be granted prestige. Competence needs to be acknowledged by others (e.g. by having many social connections), and these others must expect to benefit from that competence somehow. In our example of football players, it would be difficult for an excellent football player to be conferred prestige if he/she is never seen playing or if he/she plays well but he/she plays in a rival team or does not share advice about his/her football skills. Therefore, prestige-biased hierarchies are not only meritocratic but also beneficial for less knowledgeable/skilful individuals because being close to prestigious individuals gives them opportunities for social learning to acquire valuable knowledge/skills (Henrich \& Gil-White 2001) and receive private (Pinker 1998, p. 499) and public (Price \& Van Vugt 2014) goods.

In contrast, dominance-based hierarchies entail the ordering of individuals in the capacity to win fights or coerce and intimidate others (Redhead et al. 2018b). Dominance is a form of informal social rank that is imposed upon others (de Waal-Andrews et al. 2015; Henrich \& Gil-White 2001), sometimes with the help of coalitional alliances. Consequently, the top of dominance-based hierarchies are usually occupied by individuals who possess physical and material characteristics that are useful to win fights and intimidate others, such as having greater strength, higher fighting skills, greater coalitional support, and the possession of more and/or better weapons or other resources that could be used to inflict costs. Therefore, dominance-based hierarchies are mainly beneficial for the dominant individuals and their allies. People who do not have a dominant position tend to dislike this type of hierarchy (Brand \& Mesoudi 2019; Ridgeway \& Diekema 1989) and, if possible, try to escape the influence of dominant individuals and rebel against them (Boehm et al. 1993; Cheng 2019; Ridgeway \& Diekema 1989).

\section{Social Rank Cues}

Because prestigious and dominant individuals are associated with benefits and costs respectively in subordinates, it is important for subordinates to identify who is prestigious and who is dominant within a social group. To this end, people use social rank cues or signals that convey information about the level of prestige and dominance of other individuals. We classify social rank cues into firstorder and second-order cues (Jiménez \& Mesoudi 2019a). First-order cues are cues related to the behaviour, appearance, personality, material possessions, etc. of an individual. These can be assessed directly by the observer. Second-order cues are cues related to the behaviour of other people towards an individual and imply, therefore, a more indirect assessment of the individual by the observer.

People use prestige cues to infer the competence of an individual within a value domain and the willingness of an individual to provide benefits for the group. Examples of first-order prestige cues are being knowledgeable or skilful (Henrich \& Gil-White 2001), humble (Cheng et al. 2010), and showing altruistic behaviour towards the in-group (Halevy et al. 2012). Examples of second-order prestige cues are being paid sustained attention to by others with prolonged eye contact, being copied by others, being popular and receiving generalised voluntary deference (Henrich \& Gil-White 2001).

People use dominance cues to infer the fighting skill of an individual and their willingness to use force and intimidation to attain their goals. Examples of first-order dominance cues are being aggressive or intimidating (Henrich \& Gil-White 2001), or being arrogant, narcissistic and self-centred (Cheng et al. 2010). Examples of secondorder dominance cues are being paid attention to by others but without receiving prolonged eye contact, being obeyed by others, being disliked/unpopular, and receiving generalised coerced deference (Henrich \& Gil-White 2001).

In this study, we contrast prestige and dominance cues with medium social rank cues. People use medium social rank cues to infer that an individual is not particularly prestigious or dominant but is not at the bottom of either hierarchy. Examples of first-order medium social rank cues are having average knowledge or skill, being modest, and highly regarding the contribution of others. Examples of second-order medium social rank cues are not being paid a lot of attention by others, not being very influential within a group, and receiving deference by only a small group of close friends and relatives. 


\section{Hypotheses}

According to Henrich and Gil-White (2001), prestige-based hierarchies evolved to identify individuals with better-thanaverage fitness-enhancing knowledge/skills from whom to learn. Based on their theory, they predicted that high prestige individuals are more likely to be copied by others than low prestige individuals. This prediction has found some support (Atkisson et al. 2012; Brand et al. 2019; Chudek et al. 2012), although with limitations (see Jiménez \& Mesoudi 2019a, b). However, a key piece in the puzzle of how people learn from prestigious individuals is missing in the literature. That is, there is a lack of studies investigating the cultural transmission of prestige cues themselves. This is an important question because in order to preferentially copy prestigious individuals, it is necessary to first identify them.

If people select individuals with better-than-average knowledge/skills from whom to learn through the identification of indirect cues that convey high prestige, as Henrich and Gil-White state, then it is likely that natural selection (or potentially cultural selection; Heyes, 2018) has shaped human cognition to be more attentive to, and process and recall better, high prestige social rank cues than social rank cues conveying lower prestige. If so, descriptions of individuals displaying high prestige social rank cues would be transmitted with greater fidelity during social interactions than descriptions of individuals displaying lower prestige social rank cues.

The identification of dominant individuals who are more likely to impose costs upon others through physical aggression or other types of intimidation might also have been important in human evolution. Natural selection (or cultural selection) might therefore have shaped human cognition to be more attentive to, and process and recall better, high dominance social rank cues than social rank cues conveying lower dominance. If this is true, then descriptions of individuals displaying high dominance social rank cues would be transmitted with greater fidelity during social interactions than descriptions of individuals displaying lower dominance. Consequently, we predict that both high prestige and high dominance social rank cues are better transmitted than medium social rank cues.

Another important question is whether high dominance cues are better transmitted than high prestige social rank cues, high prestige cues better transmitted than high dominance cues, or both types of high social rank cues are similarly well transmitted. Previous research suggests that people tend to be more attentive to, recall better, and choose to transmit more, information conveying negative and threatrelated information than neutral or positive information (Bebbington et al. 2017; Blaine \& Boyer, 2018) and that people are loss aversive, i.e. they prefer not to lose a certain amount of money than to gain the same amount of money with the same probability (Kahneman \& Tversky, 1979; but see Mukherjee et al., 2017). These pieces of evidence suggest that avoiding costs might have been more important in human evolution than attaining benefits. If so, it is likely that people are more attentive to, process, and recall better high dominant social rank cues, which signal the capacity and disposition of an individual to impose costs on others, than high prestige social rank cues, which signal the capacity to provide benefits to others. More specifically, being injured is likely to have important negative fitness consequences such as impeding physical mobility and difficulties attaining resources. Therefore, high dominance cues, which convey the capacity and willingness to inflict costs upon others should be easily identified and remembered. In contrast, lacking access to the knowledge/skill of a prestigious individual might be less detrimental given the fact that other individuals of the group (e.g. kin) are likely to possess and be willing to share knowledge/skills, which, although less valuable than the knowledge/skills of the prestigious individual, are still likely to be fitness-enhancing. Consequently, we predict that high dominance cues are better transmitted than high prestige cues.

In this experiment, we created fictional descriptions of three footballers playing in three different local football teams, who were described as prestigious, dominant or medium social rank. We used a transmission chain experimental design (Bartlett 1932; Mesoudi 2007) in which participants were organised in parallel, linear chains of four participants ('cultural generations') in order to test the accumulated effect of memory biases beyond a single individual's recall. Thirty independent chains were run in total. Participants in the initial generation received and recalled the original descriptions, while the remaining participants received the information recalled by participants in the previous generation of their chain. Before collecting data for the experiment, we preregistered the following hypotheses on the Open Science Framework (OSF) website (https://osf. io/68ves):

H1: Both high prestige and high dominance cues are more accurately transmitted over experimental cultural generations than medium social rank cues.

H2: High dominance cues are more accurately transmitted over experimental cultural generations than prestige cues.

\section{Methods}

\section{Participants}

Following the procedure stated in the preregistration (https ://osf.io/68vcs), we recruited online participants through 
Prolific (www.prolific.ac;Peer et al. 2016). Using prescreening filters, we selected participants who were aged 18-60 years, were English native speakers, had British Nationality, had an approval rating on previous Prolific studies of $90 \%$ or above, and had not participated in any of our previous studies through this website.

The data was collected at four different times (one for each 'cultural generation'), between 12 and 19 November 2019. Each time we recruited 30 participants to complete one generation. Thirty chains were necessary to provide five replications of each of the six counterbalanced versions of the experimental materials (see 'Design' section). Participants were paid at a rate of $£ 6 / \mathrm{h}$ for an estimated time of completion of 15 min for generations 1 and $2(£ 1.5)$ and 10 min for generations 3 and 4 (£1).

Following the exclusion criteria stated in the preregistration, we excluded from the dataset the data of two participants who read at least one of the footballers' descriptions at a rate greater than 1080 words/min. We derived this cut-off point from a previous transmission chain experiment (Jiménez \& Mesoudi, 2020), in which participants who read the materials faster than this pace were unable to recall anything. These two excluded participants were replaced by new participants. We also excluded two other participants who inadvertently occupied the same generation in a chain for which we had already collected a response. As stated in the preregistration, the responses for participants who completed the study first were retained.

Overall, we recruited 124 participants ( 84 females, 40 males) aged $18-60(M=37.25, \mathrm{SD}=10.27)$, with 120 of these participants (82 females, 38 males) aged 18-60 $(M=37.44, \mathrm{SD}=10.25)$ included in the analysis.

\section{Materials}

Fictional descriptions of three different local footballers (John, Bill, and James) who play in different local teams (also fictional) were created as materials to transmit along the chains. These descriptions were created with the intention of describing a prestigious (John), a dominant (Bill) and a medium social rank (James) footballer, respectively. The materials were based on the dominance-prestige distinction put forward by Henrich and Gil-White (2001) and Cheng et al. (2010). Following this account, John, our prestigious footballer, was described as a competent footballer who was admired by his teammates. In contrast, Bill, our dominant footballer, was described as a violent footballer who was feared by his teammates. James, our medium social rank footballer, was described as having average football skill without evoking strong emotions. We decided to use medium social rank rather than low social rank because low social ranked individuals were likely to elicit strong emotions of pity and sympathy. Medium social rank is lacking in both these emotions and in prestige and dominance cues.

The exact descriptions of the three footballers are as follows:

\section{Prestigious footballer (John)}

Everybody in the football team admires John. He is so skilful as a football player that last year he scored the most goals in the local league. Consequently, this year members of the team unanimously voted to make him captain of the team. They also tend to copy whatever he does. At team meetings, the other members always pay careful attention to what he is saying with their eyes fixed on him. Nevertheless, he is modest about his football skills and he always takes other team members' wishes into consideration. Outside of the football team, he is also very popular. People often invite him to parties because they want to spend time with him.

\section{Dominant footballer (Bill)}

Everybody in the football team is afraid of Bill. He is so violent as a football player that last month he injured two teammates during training. This year he self-appointed himself captain without the support of any team member. Other teammates tend to obey him. At team meetings, the other members always pay careful attention to what he is saying, though usually without making eye contact with him. He thinks he is the best player and he never takes other team members' wishes into consideration. Outside of the football team, people also dislike him, but people invite him to parties because they don't want to make him angry.

\section{Medium social rank footballer (James)}

People in the football team don't have strong emotions towards James. He is an average football player in the team, scoring only a few goals last year. This year he wanted to be the captain of the team, but he only received two votes. He isn't very influential among his teammates. At team meetings, the other members of the team often don't listen to him very carefully and don't tend to fix their eyes on him for long. He is modest about his football skills, and consequently takes other teammates' perspectives in high regards. Outside the team, he is not particularly popular but he does get invited to parties held by his closest friends because they like to hang out with him.

\section{Design}

A within-chain transmission chain design with four generations per chain was used. Participants in the first generation were asked to read and then recall the original descriptions 
Table 1 Recall coding template. Propositions 4 and 11 were split into propositions 4A and 4B, and 11A and 11B, respectively, after data collection. See main text for details

\begin{tabular}{llll}
\hline Propositions & Prestigious (John) & Dominant (Bill) & Medium social rank (James) \\
\hline P1 & Is admired & Is feared & Doesn't arouse strong emotions \\
P2 & Skilful & Violent & Average skill \\
P3 & Highest goal scorer & Injured teammates & Few goals \\
P4A+P4B & A: Being captain & A: Being captain & A: Not being captain \\
& B: Being chosen/voted & B: Being self-appointed & B: Not having many votes \\
P5 & Is copied & Is obeyed & Isn't very influential \\
P6 & Is paid attention to/is listened to & Is paid attention to/is listened to & Is not paid (a lot of) attention to/is not \\
& & & listened to (very carefully) \\
P7 & Received eye contact & People avoid eye contact & Rarely prolonged eye contact \\
P8 & Is modest & He thinks he is the best (arrogant/narcis- & Is modest \\
& & sist) & Take others' perspectives in high regard \\
P9 & Takes others into consideration & Doesn't take others into consideration & (takes others into consideration) \\
& & & Isn't particularly popular \\
P10 & Is popular/liked & Is unpopular/disliked & A: is invited to parties \\
P11A P11B & A: is invited to parties & A: is invited to parties & B: by closest friends \\
& B: by the team/people & B: by the team/people & Closest friends like to hang out with him
\end{tabular}

of all three footballers (prestigious, dominant and medium social rank) given in the 'Materials' section, although without the labels denoting prestige, dominance, or medium social rank. The product of their recall was then transmitted to the next participant in their chain (second generation), who also recalled the material. This procedure was repeated until reaching the fourth generation and across 30 separate, parallel transmission chains to provide independent replications of transmission effects. The order of presentation of the three descriptions was fully counterbalanced, which resulted in six different versions of the experimental materials ( 5 replications for each version).

\section{Procedure}

Participants were first randomly assigned to one of the 30 transmission chains and provided with the following instructions: 'In the following you will read three descriptions of three football players who play in different local football teams in the UK. We would like you to read these descriptions very carefully as you will be asked some questions about each of the football players later. It is very important that you read the information at a pace that allows you full comprehension as you will be asked some questions about this information later'. Second, they read the three descriptions of the prestigious, dominant, and medium social rank footballer. Third, they were asked to provide their basic demographic information (i.e. gender, age, first language, nationality, profession and nearest city from where they live). This served as a distractor task. Fourth, they were asked to recall the descriptions of the three footballers one by one. They were given between a minimum of 1 minute and a maximum of 3 minutes to recall the description of each footballer. Lastly, participants were thanked for their participation and informed about the goal of the experiment and our hypotheses.

\section{Coding and Data Analyses}

Participants' recall accuracy was assessed by comparing their correct recall with a preregistered table (https://osf.io/ b4nqu/ $)^{1}$ containing twelve social rank cues for each description (Table 1). Examples of prestige cues are to be skilful, admired, and copied. Examples of dominance cues are to be violent, feared and obeyed. Examples of medium social rank cues are to have average skill, not to evoke strong emotions, and not to be very influential. Table 1 contains minor differences to the preregistered version. After data collection, we realised that two cues contained two cues each. For instance, 'being chosen as captain', which in the original table was considered a unique prestige cue could be divided into having the position of captain (being captain) and the process of attainment of the captainship (being chosen/ voted). The same applied to the equivalent dominance cue ('being self-appointed captain' = being captain + attaining

\footnotetext{
$1{ }^{1}$ The registration form was submitted on 11 th November 2019. The data was collected between 12 and 19 November. During data collection, we realised that the supplementary materials for the preregistration (i.e. experimental materials, table for coding recall and R script) were not attached to the preregistration form and uploaded again on 15 November 2019.
} 
Fig. 1 Raw means for the recall of information with 1.60 standard error bars (corresponding to $89 \% \mathrm{CI}$ as given in the text) plotted against generation. As predicted by $\mathrm{H} 1$, both prestige cues and dominance cues were better transmitted than medium social rank cues. Contrary to $\mathrm{H} 2$, there were no clear differences in the transmission of prestige cues and dominance cues over generations

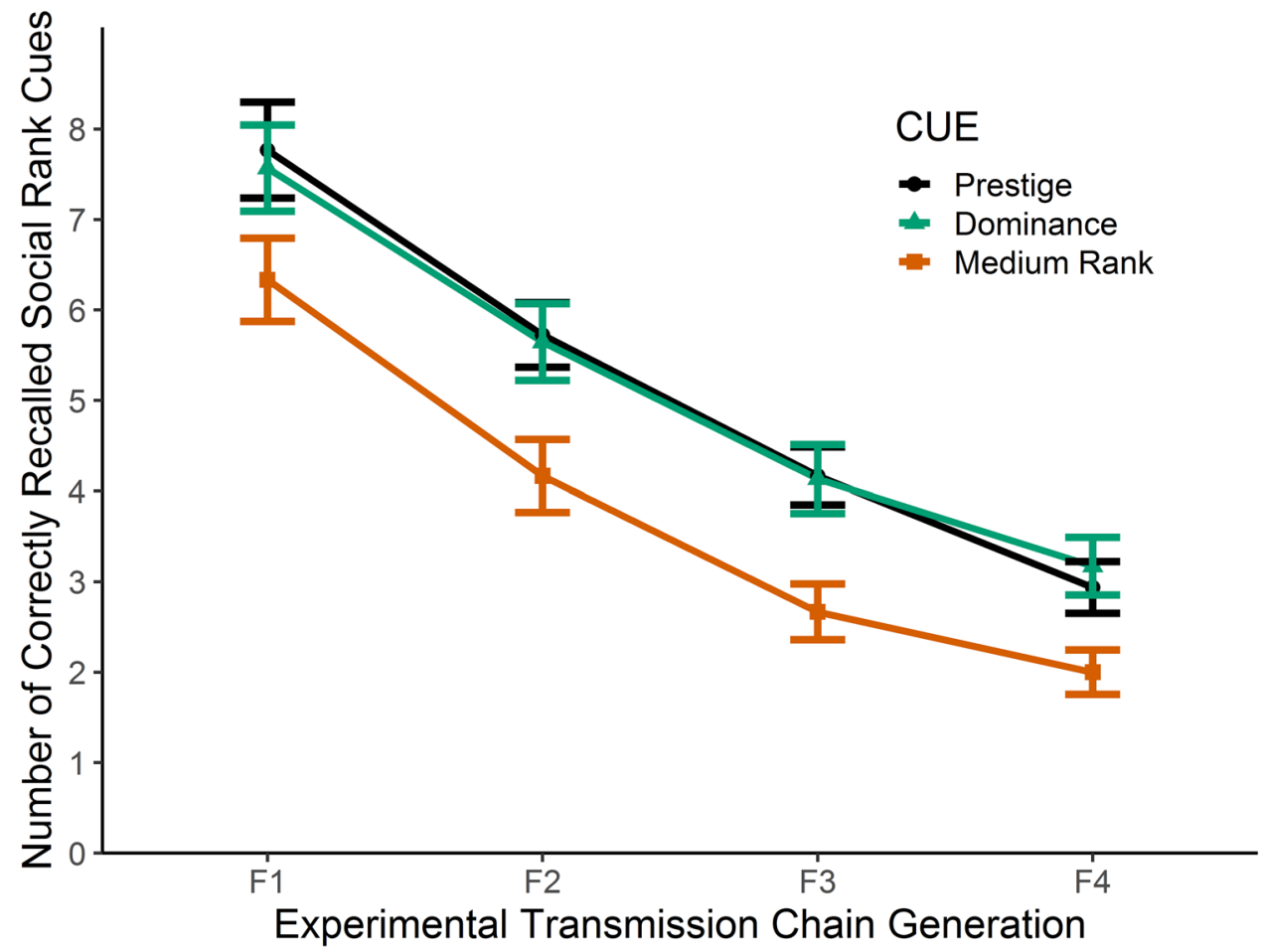

the captainship through self-appointment) and medium social rank cue ('not enough votes for being captain' = not being captain + receiving few votes). The other proposition ('being invited to parties' for both the prestigious and dominant individuals, and 'being invited to parties of closest friends' for the medium social rank individual), were considered unfair for the medium social rank individual as it contained more information to remember. Consequently, we changed the recall coding system to accept 'being invited to parties' as one cue for the three individuals. An additional proposition specified the people who invited them (i.e. only their friends for the medium social rank individual and the team or people in general for both the prestigious and dominant individual). Both coding systems (the preregistered and the new) yielded qualitatively similar results and, consequently, we only report here the results derived from the new, improved coding system. A second coder, who was blind to the hypothesis, coded $10 \%$ of the chains (3 chains) using this improved coding system. A high inter-coding reliability between both coders (Cohen's kappa $=0.86$ ) was found.

All statistical analyses were conducted with Bayesian package brms (Bürkner 2017) in R 3.5.3 (R Core and Team 2019). As in our previous transmission chain experiments (Jiménez \& Mesoudi, 2020 ; Jiménez et al. 2018), all the regression models were multilevel with intercepts varying by chain. We treated generation as a monotonic variable (Bürkner \& Charpentier 2018) as recall decreases over generations but the amount of the decrease varies between adjacent generations (Jiménez \& Mesoudi, in press). We adopted a model comparison approach, comparing a control model that included generation as the sole predictor of recall to a model that, in addition to generation, included the type of social rank cue (prestige, dominance, medium social rank) as a predictor. We use leave-one-out cross-validation information criterion (LOOIC; Vehtari et al. 2019) as a measure of relative model fit. LOOIC can be interpreted similarly to Akaike information criterion (AIC) or Watanabe-Akaike information criterion (WAIC), such that a lower LOOIC indicates better fit to the data.

\section{Results}

\section{Cumulative Recall}

Figure 1 shows the number of correctly recalled social rank cues across cultural generations for the description of each footballer (prestigious, dominant, and medium rank). As in similar transmission chain experiments, the number of correctly recalled social rank cues decreases over cultural generations. As predicted by $\mathrm{H} 1$, the figure clearly shows that the prestige and dominance cues were better transmitted than the medium social rank cues. This effect is present from the first generation, and in each subsequent generation. Contrary to $\mathrm{H} 2$, the figure does not show any clear differences in the transmission of prestige and dominance cues over generations. 
Table 2 Unstandardised coefficients $(B)$ and their standard errors (in brackets) for each of the main regression models. Square brackets indicate the reference categories for the categorical predictors. Ordinal predictors were modelled as monotonic effects and are labelled mo(variable). More regression models and further details can be found in the Supplementary Materials. LOOIC = leave-one-out cross-validation information criterion (lower values indicate better fit to the data; see text for details). Model weights were calculated using pseudo-Bayesian model average weights with Bayesian bootstrap (Vehtari \& Gabry 2019; Yao et al. 2018) with the loo package (Vehtari et al. 2019)

\begin{tabular}{llll}
\hline Unstandardised coefficients & Main regression models & & Cues $\times$ generation \\
\cline { 2 - 4 } & Generation & Cues & B (SE) \\
\hline Intercept & B (SE) & B (SE) & $1.76(0.10)$ \\
mo(Generation) & $1.92(0.08)$ & $1.70(0.09)$ & $-1.12(0.13)$ \\
Cue [Dominance] & $-0.97(0.07)$ & $-0.97(0.07)$ & $0.21(0.09)$ \\
Cue [Prestige] & & $0.30(0.06)$ & $0.25(0.08)$ \\
Cue[Dominance]:Generation & & $0.31(0.06)$ & $0.24(0.17)$ \\
Cue[Prestige]:Generation & & & $0.16(0.17)$ \\
LOOIC & 1431.3 & 1400.4 & 1400.9 \\
Model weights & 0.000 & 0.562 & 0.438 \\
\hline
\end{tabular}

To statistically analyse these trends, we produced several Bayesian multilevel Poisson regression models (see Table 2). We first ran a generation-only model that included generation as the sole predictor of recall, which was used as our control model ( $L O O I C=1431.3, \mathrm{SE}=17.4$ ). We compared this model with our two preregistered a priori models. The first was a social rank model, which included the three types of social rank cues (prestige, dominance, and medium social rank cues) together with generation as predictors of recall $(\mathrm{LOOIC}=1400.4, \mathrm{SE}=17.5)$. The second was an interaction model, which included the main effects of the social rank cues and generation together with their interaction (LOOIC $=1400.9, \mathrm{SE}=17.5)$. Both a priori models had a better fit than the control generation model, with the social rank model being slightly better than the interaction model. As predicted by $\mathrm{H} 1$, both dominance cues $(M=5.15$, $\mathrm{SD}=2.75, B=0.30, \mathrm{SE}=0.06,89 \% \mathrm{CI}[0.20,0.40])$ and prestige cues $(M=5.12, \mathrm{SD}=2.76, B=0.31, \mathrm{SE}=0.06$, $89 \%$ CI $[0.21,0.41])$ were better recalled than medium social rank cues $(M=3.79, \mathrm{SD}=2.58)$. Contrary to $\mathrm{H} 2$, the dominance cues were not better recalled than the prestige cues $(B=-0.01, \mathrm{SE}=0.06,89 \% \mathrm{CI}[-0.10,0.09])$.

Exploratory analyses were conducted to study the possible effects of gender, age, and interest in football on the transmission of social rank cues. The addition of these three variables together or separately to the social rank model did not improve its model fit (see Supplementary Materials).

\section{Narrative Evolution}

To study the narrative evolution, we analysed which social rank cues were well conserved in generation 4 for the description of the prestigious, dominant and medium social rank individuals. We also identified participants' inferences at any generation within the chains and assessed their congruency with the dominance-prestige-medium social rank distinction. These analyses were not included in the preregistration and are exploratory.

For the prestigious individual, the cue that was best conserved in generation 4 was being popular (67\%), which was followed by being invited to parties $(47 \%)$, being the team captain $(40 \%)$, being skilful playing football $(30 \%)$, and being voted for the captain position (33\%). Participants' inferences mainly referred to his good social skills (e.g. 'nice', 'friendly', 'kind', 'open'), confidence, competence (e.g. 'all round', 'golden boy'), and the wish of people to be like him. These inferences are all congruent with the notion of a prestigious individual as it was described in the introduction, i.e. as a competent, generous, and respected individual.

For the dominant individual, the cue that was best conserved was being feared $(60 \%)$, which was followed by being team captain (43\%), being invited to parties $(37 \%)$, being aggressive (33\%), having self-appointed himself the captain position $(30 \%)$, being obeyed $(30 \%)$, and being unpopular (30\%). Participants' inferences refer to him as an 'angry', 'mean', 'nasty', 'unpleasant', 'direct', 'forceful', 'hostile', 'loud' 'bully' who 'shouts a lot', 'is not a good listener', and 'people don't enjoy his company'. These inferences are congruent with the notion of a dominant individual as described in the introduction, i.e. an aggressive individual who is feared by people.

For the medium social rank individual, the cue that was best conserved was having average football skill (57\%), followed by being invited to parties (43\%). Participants' inferences refer to him as a 'good person' (also 'nice', 'pleasant', 
who 'could be trusted in times of need') with an introverted personality (e.g. 'quiet', 'shy', 'reserved' person who 'may lack confidence in larger groups'). These characteristics are compatible with the notion of medium social rank. However, opposite characteristics, i.e. being a bad person with an extroverted personality, would also be compatible with the notion of medium social rank.

\section{Discussion}

In this study, we tested whether high social rank cues (both prestige and dominance cues) are better transmitted than medium social rank cues (H1) and whether dominance cues are better transmitted than prestige cues (H2). To test these two hypotheses, we conducted a transmission chain experiment with 30 independent transmission chains each comprising four cultural generations. Supporting H1, we found that both prestige and dominance cues were better transmitted than medium social rank cues. The recall advantage of both high social rank cues was evident in the first generation, which suggests that this effect might be strong enough to be detected in single-generation experiments. This transmission advantage of the high social rank cues was maintained, but did not increase, in the subsequent generations, indicating its long-term stability over repeated transmission episodes. This result is consistent with the evolutionary importance of both prestige-based and dominance-based social hierarchies in human social groups (Henrich \& Gil-White 2001).

Contrary to $\mathrm{H} 2$, dominance cues were no better transmitted than prestige cues. We predicted a transmission advantage of dominance cues over prestige cues because we assumed that avoiding the potential costs produced by dominant individuals (e.g. physical injuries) was more important than the potential benefits provided by prestigious individuals (e.g. high-quality information to socially learn). Our experiment does not directly test this assumption, but the lack of statistical differences between the cumulative recall of prestige and dominance cues suggests that identifying both prestigious and dominant individuals might have been equally important in our evolutionary history.

Recall-based transmission chain experiments are generally used to study content transmission biases, i.e. a transmission advantage of information with particular intrinsic characteristics. These studies have identified different content biases such as transmission advantages for social (Mesoudi et al. 2006; Stubbersfield et al. 2014), emotional (Eriksson \& Coultas 2014; Stubbersfield et al. 2017), negative (Bebbington et al. 2017), and stereotypical (Kashima 2000; Lyons \& Kashima 2006) information. Our results suggest another content transmission bias to add to this list: a social rank content transmission bias. However, the results might also be explained, not by a specific content bias referring to social rank, but by a previously identified content bias relating to emotion. Stubbersfield et al. (2017) showed that narratives with high emotional content are better transmitted than narratives with low emotional content. This effect occurred independent of the valence of the emotion (positive as in amusement vs negative as in disgust). It is plausible that our descriptions of both the prestigious individual and the dominant individual have elicited a higher level of emotionality than our description of our medium social rank individual. The description of a prestigious football player might have elicited positive emotions like admiration, while the description of the dominant football player might have elicited negative emotions like fear. This possibility would be congruent with Henrich and Gil-White's dual evolutionary model of social hierarchy, which states that the emotions of admiration and fear elicited in others are the mechanisms by which prestigious and dominant individuals respectively acquired high social rank and influence. As emotions are a proximate mechanism, emotional and social rank content biases are not necessarily alternative explanations. Emotions such as admiration and fear could be the proximate mechanism by which the ultimate-level social rank bias operates. Future studies should use procedures to balance the emotional content across experimental conditions to test whether the elicitation of emotions is the mechanism by which social rank bias operates at a proximate level.

Another possibility is that the experimental materials for both prestige and dominance cues were easier to remember (i.e. less cognitively demanding) than the medium social rank cues due to their higher level of concreteness (Heath \& Heath 2008). High social rank cues tend to refer to a small subset of individuals within a group and are clearly distinguishable from lower social rank cues due to their exceptionality (e.g. highest goal scorer for prestige, violent for dominance). In contrast, medium social rank cues refer to a larger number of individuals and are difficult to distinguish from low social rank cues. Therefore, the transmission advantage of high social rank cues over medium social rank cues might be a consequence of their capacity to differentiate between people rather than identifying high social rank individuals. Future studies might use a description of an individual at the bottom of both dominance and prestige hierarchies to avoid the problem of different levels of concreteness between high social rank and lower social rank. A description of an individual at the bottom of a social hierarchy could also elicit higher level of emotions (e.g. compassion, pity) than the medium social rank description, which might help to alleviate the problem of different levels of emotional content.

Although we tried to make the descriptions of the footballers differ only in cues of prestige and dominance, in hindsight there were a few other differences in the way we described them. For example, while the prestigious and medium ranked footballers contained information about the 
number of goals they scored (i.e. cues of success), this information was absent from the dominant description. While this asymmetry between the prestigious and dominant individual could not have explained our results, given that we found no difference in the transmission fidelity of these two descriptions, future work should more carefully control or eliminate such asymmetries.

It is interesting to compare our results with those from a similar transmission chain experiment that we recently conducted in which the information to recall by participants were arguments in favour and against the replacement of textbooks by computer tablets at schools (Jiménez \& Mesoudi, 2020). These arguments were associated with sources of information with different level of prestige and relevance for the topic (Head of the Department of Education of a leading university: high prestige, high relevance; an airline pilot: high prestige, low relevance; a cleaner: low prestige, low relevance), which were artificially maintained throughout generations. That is, prestige was manipulated as a model-based transmission bias. Contrary to the prestige bias hypothesis, there were no differences in the transmission of information between the arguments associated with high and low prestige sources. In the present experiment, prestige was manipulated as a content bias and we found an advantage of high prestige social rank cues over medium social rank cues. This suggests that, even though prestige cues are memorable compared to lower-prestige cues as we have shown here, the content of the information itself might be a more important factor on the cumulative recall of information than the prestige of the source of information (see also Berl et al. 2020).

In conclusion, we have found evidence for the higher fidelity transmission of high social rank cues, referring to both prestige and dominance, than medium social rank cues. We found no evidence for a transmission advantage of dominant over prestige cues, with each type transmitted equally well. However, the mechanism that explains these results is not clear. It could be the consequence of a specific content transmission bias referring to high social rank (social rank content transmission bias) or it could be a more general feature of the information such as a higher level of emotional content or specificity of the experimental materials for both the prestigious and dominant individual than for the medium social rank individual. Given that this is the first experiment studying the cultural transmission of social rank cues, we encourage both direct and conceptual replications of this study to further explore the transmission advantage of social rank cues.

\section{Conflict of Interest}

The authors declare that they have no conflict of interest.

\section{Ethical Statement}

The study was approved by the Biosciences Ethical Committee at the University of Exeter Cornwall Campus on the 4th November 2019 (Ref eCORN002174 v3.3).

\section{Availability of data and material}

Below is the link to the dataset and preview of the experiment, and Dataset (CSV, 133KB) - process_raw_data included.csv can be found in the Electronic Supplementary Material section Preview of the experiment: https://exetercles.eu.qualtrics.com/jfe/form/SV_071cfHiCY9qBEkB..

Funding University of Exeter

Open Access This article is licensed under a Creative Commons Attribution 4.0 International License, which permits use, sharing, adaptation, distribution and reproduction in any medium or format, as long as you give appropriate credit to the original author(s) and the source, provide a link to the Creative Commons licence, and indicate if changes were made. The images or other third party material in this article are included in the article's Creative Commons licence, unless indicated otherwise in a credit line to the material. If material is not included in the article's Creative Commons licence and your intended use is not permitted by statutory regulation or exceeds the permitted use, you will need to obtain permission directly from the copyright holder. To view a copy of this licence, visit http://creativecommons.org/licenses/by/4.0/.

\section{References}

Anderson, C., Hildreth, J. A. D., \& Howland, L. (2015). Is the desire for status a fundamental human motive? A review of the empirical literature. Psychological Bulletin, 141(3), 574-601. https:// doi.org/10.1037/a0038781.

Anderson, C., \& Kilduff, G. J. (2009). Why do dominant personalities attain influence in face-to-face groups? The competencesignaling effects of trait dominance. Journal of Personality and Social Psychology, 96(2), 491-503. https://doi.org/10.1037/ a0014201.

Anderson, C., \& Willer, R. (2014). Do status hierarchies benefit groups? A bounded functionalist account of status. In J. T. Cheng, J. L. Tracy, \& C. Anderson (Eds.), The psychology of social status (pp. 47-70). New York, NY: Springer, New York.

Atkisson, C., O'Brien, M. J., \& Mesoudi, A. (2012). Adult learners in a novel environment use prestige-biased social learning. Evolutionary Psychology, 10(3), 519-537.

Bartlett, F. C. (1932). Remembering: a study in experimental and social psychology. Cambridge: Cambridge University Press.

Bebbington, K., MacLeod, C., Ellison, T. M., \& Fay, N. (2017). The sky is falling: evidence of a negativity bias in the social transmission of information. Evolution and Human Behavior, 38(1), 92-101. https://doi.org/10.1016/j.evolhumbehav.2016.07.004.

Berl, R. E. W., Samarasinghe, A. N., Roberts, S., Jordan, F., \& Gavin, M. C. (2020, August 8). Prestige and content biases 
together shape the cultural transmission of narratives. Preprint at https://doi.org/10.31235/osf.io/9jk6g

Betzig, L. (1988). Despotism and differential reproduction: A Darwinian view of history (Vol. 14).

Blader, S. L., \& Chen, Y.-R. (2014). What's in a name? Status, power, and other forms of social hierarchy. In J. T. Cheng, J. L. Tracy, \& C. Anderson (Eds.), The psychology of social status (pp. 71-95). New York, NY: Springer, New York.

Blaine, T., \& Boyer, P. (2018). Origins of sinister rumors: A preference for threat-related material in the supply and demand of information. Evolution and Human Behavior, 39(1), 67-75. https://doi.org/10.1016/j.evolhumbehav.2017.10.001.

Boehm, C., Barclay, H. B., Dentan, R. K., Dupre, M.-C., Hill, J. D., Kent, S., et al. (1993). Egalitarian behavior and reverse dominance hierarchy [and comments and reply]. Current Anthropology, 34(3), 227-254. https://doi.org/10.1086/204166.

Brand, C. O., Heap, S., Morgan, T. J., \& Mesoudi, A. (2020). The emergence and adaptive use of prestige in an online social learning task. Scientific Reports, 10 (12095)

Brand, C. O., \& Mesoudi, A. (2019). Prestige and dominance-based hierarchies exist in naturally occurring human groups, but are unrelated to task-specific knowledge. Royal Society Open Science, 6(5), 181621. https://doi.org/10.1098/rsos.181621.

Bürkner, P.-C. (2017). brms: An R package for Bayesian multilevel models using Stan. Journal of Statistical Software, 80(1), 28. https://doi.org/10.18637/jss.v080.i01

Bürkner, P.-C., \& Charpentier, E. (2018). Monotonic effects: A principled approach for including ordinal predictors in regression models. PsyArXiv. https://doi.org/10.31234/osf.io/9qkhj.

Chagnon, N. (1988). Life histories, blood revenge, and warfare in a tribal population. Science, 239(4843), 985-992. https://doi. org/10.1126/science.239.4843.985.

Cheng, J. T. (2019). Dominance, prestige, and the role of leveling in human social hierarchy and equality. Current Opinion in Psychology. https://doi.org/10.1016/j.copsyc.2019.10.004

Cheng, J. T., \& Tracy, J. L. (2014). Toward a unified science of hierarchy: Dominance and prestige are two fundamental pathways to human social rank. 3-27. https://doi. org/10.1007/978-1-4939-0867-7_1

Cheng, J. T., Tracy, J. L., Foulsham, T., Kingstone, A., \& Henrich, J. (2013). Two ways to the top: Evidence that dominance and prestige are distinct yet viable avenues to social rank and influence. Journal of Personality and Social Psychology, 104(1), 103-125. https://doi.org/10.1037/a0030398.

Cheng, J. T., Tracy, J. L., \& Henrich, J. (2010). Pride, personality, and the evolutionary foundations of human social status. Evolution and Human Behavior, 31(5), 334-347. https://doi. org/10.1016/j.evolhumbehav.2010.02.00.

Chudek, M., Heller, S., Birch, S., \& Henrich, J. (2012). Prestigebiased cultural learning: Bystander's differential attention to potential models influences children's learning. Evolution and Human Behavior, 33, 46-56.

de Waal-Andrews, W., Gregg, A. P., \& Lammers, J. (2015). When status is grabbed and when status is granted: Getting ahead in dominance and prestige hierarchies. British Journal of Social Psychology, 54(3), 445-464. https://doi.org/10.1111/bjso.12093

Eriksson, K., \& Coultas, J. C. (2014). Corpses, maggots, poodles and rats: Emotional selection operating in three phases of cultural transmission of urban legends. Journal of Cognition and Culture, 14(1-2), 1-26. https://doi.org/10.1163/15685373-12342107.

Halevy, N., Chou, E. Y., Cohen, T. R., \& Livingston, R. W. (2012). Status conferral in intergroup social dilemmas: Behavioral antecedents and consequences of prestige and dominance. Journal of Personality and Social Psychology, 102(2), 351-366. https://doi. org/10.1037/a0025515.
Heyes, C. (2018). Cognitive gadgets: The cultural evolution of thinking. Harvard University Press.

Heath, C., \& Heath, D. (2008). Made to stick: Why some ideas take hold and others come unstuck. London: Arrow Books.

Henrich, J., \& Gil-White, F. J. (2001). The evolution of prestige: Freely conferred deference as a mechanism for enhancing the benefits of cultural transmission. Evolution and Human Behavior, 22(3), 165-196. https://doi.org/10.1016/S1090-5138(00)00071-4.

Hill, J. (1984). Prestige and reproductive success in man. Evolution and Human Behavior, 5(2), 77-95. https://doi.org/10.1016/01623095(84)90011-6.

Jiménez, Á. V., \& Mesoudi, A. (2019a). Prestige-biased social learning: Current evidence and outstanding questions. Palgrave Communications, 5(1), 20. https://doi.org/10.1057/s41599-019-0228-7.

Jiménez, Á. V., \& Mesoudi, A. (2020). Prestige does not affect the cultural transmission of novel controversial arguments in an online transmission chain experiment. Journal of Cognition and Culture, 20 (3-4), 238-261. https://doi.org/10.1163/15685373-12340083

Jiménez, Á. V., \& Mesoudi, A. (2019b, December 27). The integrated dual evolutionary model of social hierarchy. Preprint at https:// psyarxiv.com/sh7mg/

Jiménez, Á. V., Stubbersfield, J. M., \& Tehrani, J. J. (2018). An experimental investigation into the transmission of antivax attitudes using a fictional health controversy. Social Science \& Medicine, 215, 23-27. https://doi.org/10.1016/j.socscimed.2018.08.032.

Kahneman, D., \& Tversky, A. (1979). Prospect theory: An analysis of decision under risk. Econometrica, 47(2), 263-291. https://doi. org/10.2307/1914185.

Kashima, Y. (2000). Maintaining cultural stereotypes in the serial reproduction of narratives. Personality and Social Psychology Bulletin, 26(5), 594-604. https://doi.org/10.1177/0146167200 267007.

Lyons, A., \& Kashima, Y. (2006). Maintaining stereotypes in communication: Investigating memory biases and coherence-seeking in storytelling. Asian Journal of Social Psychology, 9(1), 59-71. https://doi.org/10.1111/j.1467-839X.2006.00184.x.

Maner, J. K., \& Case, C. R. (2016). Dominance and prestige: Dual strategies for navigating social hierarchies. In M. O. James \& P. Z. Mark (Eds.), Advances in experimental social psychology (Vol. Volume 54, pp. 129-180): Academic Press.

Mealey, L. (1985). The relationship between social status and biological success: A case study of the Mormon religious hierarchy. Ethology and Sociobiology, 6(4), 249-257. https://doi. org/10.1016/0162-3095(85)90017-2.

Mesoudi, A. (2007). Using the methods of experimental social psychology to study cultural evolution. Journal of Social, Evolutionary, and Cultural Psychology, 1(2), 35.

Mesoudi, A., Whiten, A., \& Dunbar, R. (2006). A bias for social information in human cultural transmission. British Journal of Psychology, 97(Pt 3), 405-423. https://doi.org/10.1348/000712605x 85871.

Mukherjee, S., Sahay, A., Pammi, V. S. C., \& Srinivasan, N. (2017). Is loss-aversion magnitude-dependent? Measuring prospective affective judgments regarding gains and losses. Judgment and Decision Making, 12(1), 81-89.

Peer, E., Samat, S., Brandimarte, L., \& Acquisti, A. (2016). Beyond the Turk: an empirical comparison of alternative platforms for crowdsourcing online behavioral research. SSRN. https://doi. org/10.2139/ssrn.2594183.

Pinker, S. (1998). How the mind works. St Ives: Penguin.

Price, M. E., \& Van Vugt, M. (2014). The evolution of leader-follower reciprocity: the theory of service-for-prestige. Frontiers in Human Neuroscience, 8(363). https://doi.org/10.3389/fnhum.2014.00363

R Core Team. (2019). R: A language and environment for statistical computing. Viena: R Foundation for Statistical Computing. Retrieved from https://www.R-project.org/ 
Redhead, D., Cheng, J. T., \& O'Gorman, R. (2018a). Higher status in group. In Shackelford T, Weekes-Shackelford V (Eds.), Encyclopedia of evolutionary psychological science. New York: Springer.

Redhead, D., Cheng, J. T., \& O'Gorman, R. (2018). Individuals that Impose Costs. In T. K. Shackelford \& V. A. Weekes-Shackelford (Eds.), Encyclopedia of evolutionary psychological science. Cham: Springer International Publishing.

Ridgeway, C. L., \& Diekema, D. (1989). Dominance and collective hierarchy formation in male and female task groups. American Sociological Review, 79-93.

Savin-Williams, R. C. (1979). Dominance hierarchies in groups of early adolescents. Child Development, 50(4), 923-935. https:// doi.org/10.2307/1129316.

Smith, J. A., \& Foti, R. J. (1998). A pattern approach to the study of leader emergence. The Leadership Quarterly, 9(2), 147-160. https ://doi.org/10.1016/s1048-9843(98)90002-9.

Snyder, J. K., Kirkpatrick, L. A., \& Barrett, H. C. (2008). The dominance dilemma: Do women really prefer dominant mates? Personal Relationships, 15(4), 425-444. https://doi.org/10.111 1/j.1475-6811.2008.00208.x.

Stubbersfield, J. M., Tehrani, J. J., \& Flynn, E. G. (2014). Serial killers, spiders and cybersex: Social and survival information bias in the transmission of urban legends. British Journal of Psychology, 106(2), 288-307. https://doi.org/10.1111/bjop.12073.

Stubbersfield, J. M., Tehrani, J. J., \& Flynn, E. G. (2017). Chicken tumours and a fishy revenge: Evidence for emotional content bias in the cumulative recall of urban legends. Journal of Cognition and Culture, 17(1-2), 12-26. https://doi.org/10.1163/1568537312342189.
Vehtari, A., Gabry, J., \& Gelman, A. (2019). loo: Efficient leave-oneout cross-validation and WAIC for Bayesian models. Retrieved from https://mc-stan.org/loo

Vehtari, A., \& Gabry, J. (2019). Bayesian Stacking and Pseduo-BMA weights using the loo package. Retrieved from https://cran.r-proje ct.org/web/packages/loo/vignettes/loo2-weights.html

Von Rueden, C. (2014). The roots and fruits of social status in smallscale human societies. In The psychology of social status (pp. 179-200): Springer.

von Rueden, C., Gurven, M., \& Kaplan, H. (2010). Why do men seek status? Fitness payoffs to dominance and prestige. Proceedings of the Royal Society B: Biological Sciences. https://doi.org/10.1098/ rspb.2010.2145

von Rueden, C., \& Jaeggi, A. V. (2016). Men's status and reproductive success in 33 nonindustrial societies: Effects of subsistence, marriage system, and reproductive strategy. Proceedings of the National Academy of Sciences of the United States of America, 113(39), 10824-10829. https://doi.org/10.1073/pnas.1606800113.

Yao, Y., Vehtari, A., Simpson, D., \& Gelman, A. (2018). Using stacking to average Bayesian predictive distributions (with discussion). Bayesian Analysis, 13(3), 917-1003.

Publisher's Note Springer Nature remains neutral with regard to jurisdictional claims in published maps and institutional affiliations. 